ISSN 0258-7122 (Print), 2408-8293 (Online)

Bangladesh J. Agril. Res. 43(4): 619-630, December 2018

\title{
INTEGRATED MANAGEMENT OF TOBACCO CATERPILLAR AND CABBAGE BUTTERFLY WITH HOST PLANT RESISTANT AND ORGANIC AMENDMENT
}

\author{
S. TALUKDER ${ }^{1}$, M. M. H. KHAN ${ }^{2}$, J. FERDOUS ${ }^{3}$ AND M. O. FARUQ ${ }^{4}$
}

\begin{abstract}
Field study was conducted at Patuakhali Science and Technology University, Patuakhali during 2013-2014 to know the effect of cabbage variety and organic agriculture on the damage potential of Spodoptera litura F. and Pieris brassicae L. Two cabbage varieties viz., Atlas-70 $\left(\mathrm{V}_{1}\right)$ and Super tropic $\left(\mathrm{V}_{2}\right)$, and organic agriculture like, $\mathrm{M}_{0}=$ control, $\mathrm{M}_{1}=$ cowdung @6 kgplot $^{-1}, \mathrm{M}_{2}=$ mustard oilcake@750gplot ${ }^{-1}, \mathrm{M}_{3}=$ Vermicompost @ 3 kgplot $^{-1}$ and $\mathrm{M}_{4}=$ Trichoderma @ 3 kgplot $^{-1}$ were included in this study. Treatment combinations were $V_{1} M_{0}$, $\mathrm{V}_{1} \mathrm{M}_{1}, \mathrm{~V}_{1} \mathrm{M}_{2}, \mathrm{~V}_{1} \mathrm{M}_{3}, \mathrm{~V}_{1} \mathrm{M}_{4}, \mathrm{~V}_{2} \mathrm{M}_{0}, \mathrm{~V}_{2} \mathrm{M}_{1}, \mathrm{~V}_{2} \mathrm{M}_{2}, \mathrm{~V}_{2} \mathrm{M}_{3}$ and $\mathrm{V}_{2} \mathrm{M}_{4}$. Results revealed that the lowest number of infested plants/plot and percent infested leavesplant ${ }^{-1}$ were found in variety Super tropic and trichoderma $\left(V_{2} M_{4}\right)$ applied plots followed by variety Atlas-70 and vermicompost $\left(\mathrm{V}_{1} \mathrm{M}_{3}\right)$ applied plots on different dates of observation while the highest number of infested plantsplot ${ }^{-1}$ and percent infested leaves plant ${ }^{-1}$ were in variety Super tropic and mustard oil cake $\left(\mathrm{V}_{2} \mathrm{M}_{2}\right)$ applied plots followed by variety Atlas-70 and control $\left(\mathrm{V}_{1} \mathrm{M}_{0}\right)$ applied plots. The highest number and percent $\left(4.00\right.$ plot $\left.^{-1}\right)$ and $\left(20.00 \%\right.$ plot $\left.^{-1}\right)$ of infested heads were found in variety Super tropic and mustard oil cake $\left(\mathrm{V}_{2} \mathrm{M}_{2}\right)$ applied plots followed by variety Atlas-70 and control plots $\left(\mathrm{V}_{1} \mathrm{M}_{0}\right)$ (16.65\%). The highest number of healthy heads plot $^{-1}$ (19.67), healthy head yield (71.47 $\mathrm{t} \mathrm{ha}^{-1}$ ) and total head yield (72.14 t ha-1) were recorded in variety Super tropic and Tricoderma $\left(\mathrm{V}_{2} \mathrm{M}_{4}\right)$ applied plots followed by variety Atlas-70 and vermicompost $\left(\mathrm{V}_{1} \mathrm{M}_{3}\right)\left(70.53 \mathrm{t} \mathrm{ha}^{-1}\right)$ applied plots while the lowest number of healthy heads per plot, healthy head yield and total head yield were recorded in in variety Super tropic and mustard oil cake $\left(\mathrm{V}_{2} \mathrm{M}_{2}\right)$ applied plots followed by variety Atlas-70 and control plots $\left(\mathrm{V}_{1} \mathrm{M}_{0}\right)$.
\end{abstract}

Keywords: Atlas-70, organic materials, trichoderma, Super tropic, vermicompost.

\section{Introduction}

Cabbage (Brassica oleracea L. var. capitata) is one of the most popular vegetables in the world. It is mostly grown in winter throughout Bangladesh. In Bangladesh, the annual production of cabbage is 217 thousand tons (BBS, 2014). The production and yield of cabbage is greatly hampered by many insect pests. Cabbage is also known to be infested by several insect pests viz., tobacco caterpillar, Spodoptera litura (Fab.), cabbage butterfly (Pieris brassicae),

${ }^{1,2 \& 4}$ Department of Entomology, Patuakhali Science and Technology University, Dumki, Patuakhali-8602, ${ }^{3}$ Scientific Officer, Bangladesh Jute Research Institute, Dhaka, Bangladesh. 
diamond back moth (Plutella xylostella Linnaeus) and cabbage aphid (Brevicoryne brassicae L.). Out of these, cabbage butterfly, diamond back moth and tobacco caterpillar are the most destructive pests causing severe yield loss to cabbage every year (Rao and Lal, 2005; Mahla et al., 2005; Kumar et al., 2007). Tobacco caterpillar is the most destructive insect pest sometimes cause complete failure of the crop. After hatching, the caterpillars start feeding on the under surface of the leaves. Leaves of heavily damaged plants have many feeding holes and sometimes the leaves take a 'sieve-like' appearance. It also destroys the leaves of cabbage by making holes in the head and greatly reduces its market value. As a result of feeding, the plants either fail to form compact cabbage heads or produce deformed heads (Uddin et al., 2007).

The indirect effects of fertilization practices acting through changes in the nutrient composition of the crop have been reported to influence plant resistance to many insect pests. Vermicompost are produced through the interactions between earthworms and microorganism in the breakdown of organic wastes and to convert into nutritional rich humus (Sinha et al., 2010). The vermicompost promote growth from $50-100 \%$ over conventional compost and $30-40 \%$ over chemical fertilizers (Sinha et al., 2010). Crop losses due to insects and diseases are reduced with organic farming (Merrill, 1983). Vermicomposts are rich in humic acid and phenolic compounds. Phenolic compounds act as feeding deterrents and hence significantly influence pest infestation (Mahanil et al., 2008; Bhonwong et al., 2009). Stevenson et al. (1993) reported that inhibition of $S$. litura development by phenolic compound from the wild groundnut. Meyer (2000) argues that soil nutrient availability not only affects the amount of damage that plants receive from herbivores but also increase the ability of plants to recover from herbivory; however, these two factors are rarely considered together. Describing the effects of soil fertility on both the degree of defoliation and compensation for herbivory for Brassica nigra plants damaged by Pieris rapae caterpillars (Meyer, 2000). Keeping these views in mind, the present study was conducted to evaluate the combine effect of varieties of cabbage and organic agriculture on the damage potential of $S$. litura and $P$. brassicae infesting cabbage.

\section{Materials and Methods}

The study was conducted at the agricultural farm of Patuakhali Science and Technology University, Dumki, Patuakhali during rabi season of 2013-2014. Two cabbage varieties viz., Atlas- $70\left(\mathrm{~V}_{1}\right)$ and Super tropic $\left(\mathrm{V}_{2}\right)$ were included in this study. Organic agricultural components were used as treatments were $\mathrm{M}_{0}=$ control, $\mathrm{M}_{1}=$ cowdung @6 kgplot ${ }^{-1}, \mathrm{M}_{2}=$ mustard oilcake @ 750gplot ${ }^{-1}, \mathrm{M}_{3}=$ Vermicompost @3 kgplot ${ }^{-1}$ and $\mathrm{M}_{4}=$ Trichoderma @ 3 kgplot $^{-1}$. Treatment combinations were $\mathrm{V}_{1} \mathrm{M}_{0}, \mathrm{~V}_{1} \mathrm{M}_{1}, \mathrm{~V}_{1} \mathrm{M}_{2}, \mathrm{~V}_{1} \mathrm{M}_{3}, \mathrm{~V}_{1} \mathrm{M}_{4}, \mathrm{~V}_{2} \mathrm{M}_{0}, \mathrm{~V}_{2} \mathrm{M}_{1}, \mathrm{~V}_{2} \mathrm{M}_{2}, \mathrm{~V}_{2} \mathrm{M}_{3}$ and $\mathrm{V}_{2} \mathrm{M}_{4}$. Experiment was laid out in randomized complete block design (RCBD) with three replications. The entire field was divided into three blocks and each block was again divided into ten plots. The distance between both 
blocks and plots was $1.0 \mathrm{~m}$. The area of each experimental plot was $9 \mathrm{~m}^{2}$. Seeds of the selected cabbage cultivars were sown in the month of November and were transplanted in December. Thirty day-old cabbage seedlings were transplanted in the plot of $9 \mathrm{~m}^{2}$ area with $45 \mathrm{~cm} \times 45 \mathrm{~cm}$ spacing on 29 December, 2013. The chemical fertilizers viz., urea, TSP and MP were applied at the rate of 285, 145 and $218 \mathrm{kgha}^{-1}$. Fertilizer, irrigation and all other agronomic practices were carried out in the experimental field whenever necessary. Harvesting of cabbage heads were started on 02 February and continued till 28 February 2014. Weekly observations were done since one week of transplanting till maturity of the crop. The number of infested plants plot $^{-1}$, the number infested leaves plant $^{-1}$ and infested head were separated carefully and healthy and infested leaves were counted and recorded. After each harvest, the number of healthy and infested heads were sorted, counted and recorded separately. The weight of healthy and infested heads per plot was recorded separately for each treatment replicates. Treatment wise percent head infestation was calculated from the pooled data of ten observations. The total yield of cabbage $\mathrm{ha}^{-1}$ for each treatment was calculated in tons from cucmulative head harvested from a plot. Data were analyzed after appropriate transformation following RCBD using computer MSTAT C and MS excel programs. Means were compared by DMRT.

\section{Results and Discussion}

Combined effects of cabbage varieties and organic agriculture on the number of infested plants plot $^{-1}$ varied significantly on different dates of observations (Table 1). On $8^{\text {th }}$ January, the number of infested plants plot $^{-1}$ ranged from $1.50-3.50$. The lowest number of infested plants plot $^{-1}$ was 1.50 for the treatment combination of $\mathrm{V}_{2} \mathrm{M}_{4}$ (Super tropic + trichoderma) which was statistically identical to $\mathrm{V}_{1} \mathrm{M}_{1}$ (1.50) and $\mathrm{V}_{2} \mathrm{M}_{1}$ (1.50). The highest number of infested plant plot $^{-1}$ was 3.50 for the treatment combination of $\mathrm{V}_{2} \mathrm{M}_{2}$ (Super tropic + mustard oil cake) which was statistically similar to $\mathrm{V}_{2} \mathrm{M}_{0}$ (Super tropic + control).

On 15 January, the number of infested plants plot $^{-1}$ ranged from 1.00 to 3.50 . The lowest number of infested plants plot ${ }^{-1}$ was recorded for the treatment combination of $\mathrm{V}_{1} \mathrm{M}_{3}$ (var. Atlas + vermicompost) which were statistically similar to $\mathrm{V}_{2} \mathrm{M}_{4}$ (Super tropic + trichoderma). The highest number of infested plant plot $^{-1}$ was for variety Super tropic and mustard oil cake $\left(V_{2} M_{2}\right)$ applied plots which were statistically similar to $\mathrm{V}_{2} \mathrm{M}_{0}$ (Super tropic and control).

On day 22 June 2014, the number of infested plants plot $^{-1}$ ranged from 0.50 to 3.00. Significantly the lowest number of infested plants plot $^{-1}$ was for the treatment combination of $\mathrm{V}_{1} \mathrm{M}_{3}$ (Atlas + vermicompost). The higher number of infested plants plot ${ }^{-1}$ was recorded in $\mathrm{V}_{2} \mathrm{M}_{1}$ (Super tropic + cowdung) which was statistically similar to remaining all other treatment combinations.

On 29 January 2014, no significant differences were observed among different treatment combinations in respect of the number of infested plants plot $^{-1}$ (Table 1 ).

On 5 February 2014, number of infested plants plot ${ }^{-1}$ ranged from 0.0 to 3.00. No plant was infested in treatment combinations of $\mathrm{V}_{2} \mathrm{M}_{4}$ (Super tropic + 
trichoderma) and the lowest number was recorded in $\mathrm{V}_{1} \mathrm{M}_{4}$ (0.50) (Atlas + trichoderma) applied plots. The higher number of infested plants/plot was in $\mathrm{V}_{1} \mathrm{M}_{0}$ applied plots which was statistically identical to $\mathrm{V}_{1} \mathrm{M}_{1}$ (Atlas + cowdung) (3.00) but statistically similar to treatment combinations of $V_{1} M_{0} V_{1} M_{2}$ on the same date.

On 12 February 2014, the number of infested plants plot $^{-1}$ ranged from 2.00 to 6.50. The lowest number of infested plants plot $^{-1}$ was observed in variety supertropic and trichoderma $\left(\mathrm{V}_{2} \mathrm{M}_{4}\right)$ applied plots which was statistically identical to $\mathrm{V}_{1} \mathrm{M}_{4}$ (2.00) and $\mathrm{V}_{2} \mathrm{M}_{0}(2.00)$ applied plots. The higher number of infested plants/plot was found in variety Atlas-70 and mustard oil cake $\left(\mathrm{V}_{1} \mathrm{M}_{2}\right)$ applied plots which was statistically similar to $\mathrm{V}_{1} \mathrm{M}_{1}$ (5.50) i.e., variety Atlas -70 and cowdung plot followed by $\mathrm{V}_{1} \mathrm{M}_{0}$ (5.00) i.e., variety Atlas-70 and untreated control plot (Table 1).

Table 1. Combined effects of cabbage varieties and organic manures on the number of infested plants plot ${ }^{-1}$ by $S$. litura and $P$. brassicae on different dates of observations

\begin{tabular}{|c|c|c|c|c|c|c|c|}
\hline \multirow{2}{*}{$\begin{array}{c}\text { Treatment } \\
\text { combinations }\end{array}$} & \multicolumn{6}{|c|}{ Number of infested plants plot ${ }^{-1}$ observed on } & \multirow{2}{*}{$\begin{array}{c}\text { Average } \\
\text { number of } \\
\text { infested } \\
\text { plants plot }^{-1}\end{array}$} \\
\hline & 8 Jan. & 15 Jan. & 22 Jan. & 29 Jan. & 05 Feb. & 12 Feb. & \\
\hline $\mathrm{V}_{1} \mathrm{M}_{0}$ & $2.50 \mathrm{ab}$ & $2.50 \mathrm{~b}$ & $1.50 \mathrm{~b}$ & 2.00 & $3.00 \mathrm{a}$ & $5.00 \mathrm{~b}$ & 2.75 \\
\hline $\mathrm{V}_{1} \mathrm{M}_{1}$ & $1.50 \mathrm{c}$ & $2.50 \mathrm{~b}$ & $2.50 \mathrm{a}$ & 2.50 & $3.00 \mathrm{a}$ & $5.50 \mathrm{ab}$ & 2.92 \\
\hline $\mathrm{V}_{1} \mathrm{M}_{2}$ & $2.00 \mathrm{~b}$ & $2.00 \mathrm{c}$ & $2.50 \mathrm{a}$ & 2.00 & $2.50 \mathrm{a}$ & $6.50 \mathrm{a}$ & 2.92 \\
\hline $\mathrm{V}_{1} \mathrm{M}_{3}$ & $2.50 \mathrm{ab}$ & $1.00 \mathrm{~d}$ & $0.50 \mathrm{c}$ & 0.50 & $0.00 \mathrm{~d}$ & 2.50de & 1.67 \\
\hline $\mathrm{V}_{1} \mathrm{M}_{4}$ & $2.00 \mathrm{~b}$ & $2.50 \mathrm{~b}$ & $2.50 \mathrm{a}$ & 1.50 & $0.50 \mathrm{~cd}$ & $2.00 \mathrm{e}$ & 1.83 \\
\hline $\mathrm{V}_{2} \mathrm{M}_{0}$ & $3.00 \mathrm{a}$ & $3.00 \mathrm{a}$ & $1.50 \mathrm{~b}$ & 1.00 & $1.00 \mathrm{c}$ & $2.00 \mathrm{e}$ & 1.92 \\
\hline $\mathrm{V}_{2} \mathrm{M}_{1}$ & $1.50 \mathrm{c}$ & $2.50 \mathrm{~b}$ & $3.00 \mathrm{a}$ & 1.00 & $2.00 \mathrm{~b}$ & $3.00 \mathrm{~d}$ & 2.17 \\
\hline $\mathrm{V}_{2} \mathrm{M}_{2}$ & $3.50 \mathrm{a}$ & $3.50 \mathrm{a}$ & $3.00 \mathrm{a}$ & 1.50 & $2.50 \mathrm{a}$ & $4.00 c$ & 3.00 \\
\hline $\mathrm{V}_{2} \mathrm{M}_{3}$ & $2.50 \mathrm{ab}$ & $2.00 \mathrm{c}$ & $3.00 \mathrm{a}$ & 2.00 & $1.50 \mathrm{bc}$ & $3.50 \mathrm{~cd}$ & 2.42 \\
\hline $\mathrm{V}_{2} \mathrm{M}_{4}$ & $1.50 \mathrm{c}$ & $1.50 \mathrm{~cd}$ & $1.00 \mathrm{~b}$ & 0.50 & $0.00 \mathrm{~d}$ & $2.00 \mathrm{e}$ & 1.08 \\
\hline LSD & 0.502 & 0.499 & 0.610 & - & 0.562 & 0.611 & - \\
\hline $\begin{array}{l}\text { Level of } \\
\text { significance }\end{array}$ & $* *$ & $* *$ & $* *$ & NS & $* *$ & $* *$ & - \\
\hline CV (\%) & 4.75 & 3.80 & 3.76 & 6.48 & 1.65 & 5.40 & - \\
\hline
\end{tabular}

Values are averages of 3 replications

Means within a column followed by different letters are significantly different from each other at $5 \%(*)$ and $1 \%(* *)$ level of probability by DMRT

$\mathrm{V}_{1}=$ var. Atlas-70; $\mathrm{V}_{2}=$ Super tropic;

$\mathrm{M}_{0}=$ control, $\mathrm{M}_{1}=$ cowdung @6 kg plot ${ }^{-1}, \mathrm{M}_{2}=$ mustard oilcake @750g plot ${ }^{-1}, \mathrm{M}_{3}=$ Vermicompost @ 3 kg plot ${ }^{-1}$ and $\mathrm{M}_{4}=$ Trichoderma @ 3 kg plot ${ }^{-1}$ 
On day 8 January 2014, the percentage of infested leaves plant ${ }^{-1}$ varied from $7.79 \%$ to $58.69 \%$ but significantly the lowest percenatge of infested leaves plant ${ }^{-1}$ was recorded in variety Super tropic and trichoderma $\left(\mathrm{V}_{2} \mathrm{M}_{4}\right)$ applied plots and the highest percentage of infested leaves plant ${ }^{-1}$ was observed in variety Super tropic and mustard oil cake $\left(\mathrm{V}_{2} \mathrm{M}_{2}\right)$ applied plots (Table 2).

On 15 January 2014, significantly the lowest percenatge of infested leaves plant ${ }^{-1}$ (4.17\%) was recorded in variety Super tropic and trichoderma $\left(\mathrm{V}_{2} \mathrm{M}_{4}\right)$ applied plots and the highest percentage of infested leaves plant ${ }^{-1}$ (66.67\%) was observed in variety Atlas-70 and untreated control plot $\left(\mathrm{V}_{1} \mathrm{M}_{0}\right)$

On 22 January 2014, the lowest percenatge of infested leaves plant ${ }^{-1}$ (3.34\%) was recorded in variety Super tropic and trichoderma $\left(\mathrm{V}_{2} \mathrm{M}_{4}\right)$ applied plots which was statistically similar to $\mathrm{V}_{2} \mathrm{M}_{3}(3.57 \%)$ applied plots. The highest percentage of infested leaves plant $^{-1}$ (61.61\%) was observed in variety Super tropic and mustard oil cake $\left(\mathrm{V}_{2} \mathrm{M}_{2}\right)$ applied plots which was statistically similar to $\mathrm{V}_{1} \mathrm{M}_{0}$ (58.93\%) (Atlas-70 + untreated control plot).

On 29 January 2014, significantly the lowest percenatge of infested leaves plant ${ }^{-1}$ (4.17\%) was obtained in variety Atlas-70 and vermicompost applied plots and the highest (53.96\%) was observed in variety Super tropic and mustard oil cake $\left(\mathrm{V}_{2} \mathrm{M}_{2}\right)$ applied plots.

On 5 February 2014, significantly the highest percentage of infested leaves plant ${ }^{1}(61.67 \%)$ was observed in variety super tropic and mustard oil cake $\left(\mathrm{V}_{2} \mathrm{M}_{2}\right)$ applied plots which differed significantly from all other remaining treatments. The lowest percenatge of infested leaves plant ${ }^{-1}(3.57 \%)$ was recorded in variety Atlas-70 and trichoderma $\left(\mathrm{V}_{1} \mathrm{M}_{4}\right)$ applied plots (Table 2). No leaf infestation was observed in variety Atlas-70 and vermicompost $\left(\mathrm{V}_{1} \mathrm{M}_{3}\right)(0.00 \%)$ and variety Super tropic and trichoderma $\left(\mathrm{V}_{2} \mathrm{M}_{4}\right)(0.00 \%)$ applied plots.

On 12 February 2014, the lowest percenatge of infested leaves plant ${ }^{-1}(18.18 \%)$ was recorded in variety Atlas-70 and vermicompost applied plots which was statistically similar to $\mathrm{V}_{1} \mathrm{M}_{1}$ (22.35\%). The highest percentage of infested leaves plant $^{-1}$ (76.93\%) was observed in variety Super tropic and mustard oil cake $\left(\mathrm{V}_{2} \mathrm{M}_{2}\right)$ applied plots followed by variety Super tropic and cowdung $\left(\mathrm{V}_{2} \mathrm{M}_{1}\right)$ (62.15\%) applied plots (Table 3). No significant differences were observed among variety Atlas-70 and control plots $\left(\mathrm{V}_{1} \mathrm{M}_{0}\right)$ (55.77\%), $\mathrm{V}_{1} \mathrm{M}_{2}(54.95 \%)$ and $\mathrm{V}_{2} \mathrm{M}_{0}$ (51.25\%) applied plots (Table 2). 
Table 2. Combined effects of cabbage varieties and organic manures on the percentage of infested leaves plant ${ }^{-1}$ caused by $S$. litura and $P$. brassicae on different dates of observations

\begin{tabular}{|c|c|c|c|c|c|c|c|}
\hline \multirow{2}{*}{$\begin{array}{l}\text { Treatment } \\
\text { combinations }\end{array}$} & \multicolumn{6}{|c|}{ Percentage of infested leaves plant ${ }^{-1}$ on } & \multirow{2}{*}{$\begin{array}{l}\text { Mean } \\
\text { percentage of } \\
\text { infested } \\
\text { leaves plant }{ }^{-1}\end{array}$} \\
\hline & $8 \mathrm{Jan}$. & 15 Jan. & 22 Jan. & 29 Jan. & 05 Feb. & 12 Feb. & \\
\hline $\mathrm{V}_{1} \mathrm{M}_{0}$ & $51.12 b$ & $66.67 a$ & $58.93 a$ & $46.88 b$ & $36.64 b$ & $55.77 \mathrm{c}$ & 52.05 \\
\hline $\mathrm{V}_{1} \mathrm{M}_{1}$ & $31.45 \mathrm{~d}$ & $36.67 \mathrm{c}$ & 28.57d & $36.36 \mathrm{c}$ & $39.56 b$ & $22.35 f$ & 32.08 \\
\hline $\mathrm{V}_{1} \mathrm{M}_{2}$ & $40.41 c$ & $43.69 b$ & $34.88 \mathrm{c}$ & $39.74 c$ & 29.12c & $54.95 c$ & 40.45 \\
\hline $\mathrm{V}_{1} \mathrm{M}_{3}$ & $12.78 \mathrm{f}$ & $25.00 \mathrm{e}$ & $19.23 \mathrm{e}$ & $4.17 \mathrm{~g}$ & $0.00 \mathrm{e}$ & $18.18 \mathrm{f}$ & 13.05 \\
\hline $\mathrm{V}_{1} \mathrm{M}_{4}$ & $17.45 \mathrm{e}$ & $11.31 \mathrm{~g}$ & $15.11 \mathrm{f}$ & $17.86 \mathrm{e}$ & 3.57d & 43.34d & 17.85 \\
\hline $\mathrm{V}_{2} \mathrm{M}_{0}$ & $36.14 c$ & $31.22 \mathrm{~d}$ & $30.77 d$ & 34.38cd & $36.36 b$ & $51.25 c$ & 36.47 \\
\hline $\mathrm{V}_{2} \mathrm{M}_{1}$ & $40.36 c$ & $42.86 \mathrm{~b}$ & $41.19 \mathrm{~b}$ & 20.83e & $38.92 b$ & $62.15 b$ & 40.78 \\
\hline $\mathrm{V}_{2} \mathrm{M}_{2}$ & $58.69 a$ & $46.88 \mathrm{~b}$ & $61.61 a$ & 53.96a & $61.67 a$ & 76.93a & 59.45 \\
\hline $\mathrm{V}_{2} \mathrm{M}_{3}$ & $30.19 d$ & $17.86 f$ & $3.57 \mathrm{~g}$ & 27.09d & $39.74 b$ & $41.67 d$ & 31.07 \\
\hline $\mathrm{V}_{2} \mathrm{M}_{4}$ & $7.79 \mathrm{~g}$ & $4.17 \mathrm{~h}$ & $3.34 \mathrm{~g}$ & $7.69 \mathrm{f}$ & $0.00 \mathrm{e}$ & $31.47 \mathrm{e}$ & 8.59 \\
\hline LSD & 4.40 & 3.84 & 2.62 & 3.61 & 3.12 & 4.30 & - \\
\hline $\begin{array}{l}\text { Level of } \\
\text { significance }\end{array}$ & $* *$ & $* *$ & $*$ & $* *$ & $*$ & $*$ & - \\
\hline CV (\%) & 8.52 & 7.31 & 4.80 & 5.90 & 6.74 & 5.12 & - \\
\hline
\end{tabular}

Values are averages of 3 replications

Means within a column followed by different letters are significantly different from each other at $5 \%(*)$ and $1 \%(* *)$ level of probability by DMRT

$\mathrm{V}_{1}=$ var. Atlas-70; $\mathrm{V}_{2}=$ Supertropic;

$\mathrm{M}_{0}=$ control, $\mathrm{M}_{1}=$ cowdung @ $6 \mathrm{~kg}$ plot $^{-1}, \mathrm{M}_{2}=$ mustard oilcake @ 750g plot ${ }^{-1}, \mathrm{M}_{3}=$ Vermicompost@3 kg plot ${ }^{-1}$ and $\mathrm{M}_{4}=$ Trichoderma @ $3 \mathrm{~kg} p l o t^{-1}$

Significantly the highest number of healthy heads $\operatorname{plot}^{-1}$ (19.67) was found in variety Super tropic and trichoderma $\left(\mathrm{V}_{2} \mathrm{M}_{4}\right)$ applied plots which was statistically similar to $\mathrm{V}_{1} \mathrm{M}_{3}$ (19.33) (Atlas-70 + vermicompost). The lowest healthy heads (16.00) was in variety supertropic and mustard oil cake $\left(\mathrm{V}_{2} \mathrm{M}_{2}\right)$ applied plots which was statistically similar to $\mathrm{V}_{1} \mathrm{M}_{0}$ (16.67) (Table 3). On the contrary, the lowest number of infested heads plot $^{-1}(0.33)$ was observed in variety Super tropic and trichoderma $\left(\mathrm{V}_{2} \mathrm{M}_{4}\right)$ applied plots which was statistically similar to $\mathrm{V}_{1} \mathrm{M}_{3}$ (0.67) (Atlas-70 + vermicompost) applied plots. The highest number of infested heads per plot (4.00) was found in variety Super tropic and mustard oil cake $\left(\mathrm{V}_{2} \mathrm{M}_{2}\right)$ applied plots followed by variety Atlas-70 and control plots $\left(\mathrm{V}_{1} \mathrm{M}_{0}\right)$ (3.33) and variety Atlas-70 and mustard oil cake $\left(\mathrm{V}_{1} \mathrm{M}_{2}\right)$ (2.67) applied plots. No 
significant differences were observed among variety Atlas -70 and cowdung $\left(\mathrm{V}_{1} \mathrm{M}_{1}\right)$ (2.33), variety Super tropic and control $\left(\mathrm{V}_{2} \mathrm{M}_{0}\right)$ (2.00), variety Super tropic and cowdung $\left(\mathrm{V}_{2} \mathrm{M}_{1}\right)$ (2.12) and variety supertropic and vermicompost $\left(\mathrm{V}_{2} \mathrm{M}_{3}\right)$ (1.67) applied plots in respect of number of infested heads per plot. In case of percent head infestation by number, the lowest percent (1.65\%) was recorded in variety Super tropic and trichoderma $\left(\mathrm{V}_{2} \mathrm{M}_{4}\right)$ applied plots followed by $\mathrm{V}_{1} \mathrm{M}_{3}(3.35 \%)$ and $\mathrm{V}_{1} \mathrm{M}_{4}(6.65 \%)$ applied plots. The highest percent (20.00\%) head infestation by number was recorded in variety Super tropic and mustard oil cake $\left(\mathrm{V}_{2} \mathrm{M}_{2}\right)$ applied plots followed by variety Atlas-70 and control plots $\left(\mathrm{V}_{1} \mathrm{M}_{0}\right)$ (16.65\%). No significant difference was observed among variety Atlas -70 and cowdung $\left(\mathrm{V}_{1} \mathrm{M}_{1}\right)(11.65 \%)$, variety Super tropic and cowdung $\left(\mathrm{V}_{2} \mathrm{M}_{1}\right)(10.60 \%)$, variety Super tropic and control $\left(\mathrm{V}_{2} \mathrm{M}_{0}\right)(10.00 \%)$ in respect of the percentage of infested heads by number (Table 3 ).

Table 3. Mean number of healthy and infested head plot $^{-1}$ and percent head infestation (by number) caused by $S$. litura and $P$. brassicae as influenced by treatment combinations

\begin{tabular}{l|c|c|c}
\hline $\begin{array}{c}\text { Treatment } \\
\text { combinations }\end{array}$ & $\begin{array}{c}\text { No. of healthy heads } \\
\text { plot }^{-1}\end{array}$ & $\begin{array}{c}\text { No. of infested } \\
\text { heads } \\
\text { plot }^{-1}\end{array}$ & $\begin{array}{c}\text { Percent head } \\
\text { infestation by } \\
\text { number }\end{array}$ \\
\hline $\mathrm{V}_{1} \mathrm{M}_{0}$ & $16.67 \mathrm{e}$ & $3.33 \mathrm{ab}$ & $16.65 \mathrm{ab}$ \\
$\mathrm{V}_{1} \mathrm{M}_{1}$ & $17.67 \mathrm{~cd}$ & $2.33 \mathrm{~cd}$ & $11.65 \mathrm{~cd}$ \\
$\mathrm{~V}_{1} \mathrm{M}_{2}$ & $17.33 \mathrm{~d}$ & $2.67 \mathrm{bc}$ & $13.35 \mathrm{bc}$ \\
$\mathrm{V}_{1} \mathrm{M}_{3}$ & $19.33 \mathrm{ab}$ & $0.67 \mathrm{f}$ & $3.35 \mathrm{fg}$ \\
$\mathrm{V}_{1} \mathrm{M}_{4}$ & $18.67 \mathrm{~b}$ & $1.33 \mathrm{e}$ & $6.65 \mathrm{ef}$ \\
$\mathrm{V}_{2} \mathrm{M}_{0}$ & $18.00 \mathrm{bc}$ & $2.00 \mathrm{cde}$ & $10.00 \mathrm{cde}$ \\
$\mathrm{V}_{2} \mathrm{M}_{1}$ & $17.88 \mathrm{bd}$ & $2.12 \mathrm{cde}$ & $10.60 \mathrm{~cd}$ \\
$\mathrm{~V}_{2} \mathrm{M}_{2}$ & $16.00 \mathrm{e}$ & $4.00 \mathrm{a}$ & $20.00 \mathrm{a}$ \\
$\mathrm{V}_{2} \mathrm{M}_{3}$ & $18.33 \mathrm{bc}$ & $1.67 \mathrm{de}$ & $8.35 \mathrm{de}$ \\
$\mathrm{V}_{2} \mathrm{M}_{4}$ & $19.67 \mathrm{a}$ & $0.33 \mathrm{f}$ & $1.65 \mathrm{~g}$ \\
\hline LSD & 0.874 & 0.854 & 4.472 \\
\hline
\end{tabular}

$\mathrm{V}_{1}=$ var. Atlas-70; $\mathrm{V}_{2}=$ Super tropic;

$\mathrm{M}_{0}=$ control, $\mathrm{M}_{1}=$ cowdung @ $6 \mathrm{~kg}_{\mathrm{plot}}{ }^{-1}, \mathrm{M}_{2}=$ mustard oilcake @ 750g $\mathrm{plot}^{-1}, \mathrm{M}_{3}=$ Vermicompost @ $3 \mathrm{~kg} \mathrm{plot}^{-1}$ and $\mathrm{M}_{4}=$ Trichoderma @ $3 \mathrm{~kg} \mathrm{plot}^{-1}$

Head yield of cabbage varied significantly among different treatment combinations applied in cabbage field under the present trial. The highest healthy head yield (71.47 ton ha ${ }^{-1}$ ) was recorded in variety Super tropic and trichoderma $\left(\mathrm{V}_{2} \mathrm{M}_{4}\right)$ applied plots which was statistically similar to $\mathrm{V}_{1} \mathrm{M}_{3}\left(69.23\right.$ ton ha $\left.{ }^{-1}\right)$ applied plots but significantly different from all other treatment combinations 
while the lowest head yield (50.40 ton ha ${ }^{-1}$ ) was recorded in variety Super tropic and mustard oil cake $\left(\mathrm{V}_{2} \mathrm{M}_{2}\right)$ treated plot. However, no significant difference was observed among variety Super tropic and untreated control $\left(\mathrm{V}_{2} \mathrm{M}_{0}\right)$ (59.20 ton ha$\left.{ }^{1}\right)$ and variety Super tropic and cowdung $\left(\mathrm{V}_{2} \mathrm{M}_{1}\right)\left(58.78\right.$ ton ha $\left.{ }^{-1}\right)$ treated plots in respect of healthy head yield by weight (Table 4). In contrast, the highest infested head yield (8.11 ton ha ${ }^{-1}$ ) was recorded in variety Super tropic plus mustard oil cake $\left(V_{2} \mathrm{M}_{2}\right)$ applied plots followed by variety Atlas-70 plus control plots $\left(\mathrm{V}_{1} \mathrm{M}_{0}\right)$ (6.13 ton ha ${ }^{-1}$ ). No significant differences were observed among variety Atlas -70 plus cowdung $\left(\mathrm{V}_{1} \mathrm{M}_{1}\right)$ (4.22 ton ha $\left.{ }^{-1}\right)$, variety Super tropic plus cowdung $\left(\mathrm{V}_{2} \mathrm{M}_{1}\right)$ (4.21 ton ha $\left.{ }^{-1}\right)$, variety super tropic plus control $\left(\mathrm{V}_{2} \mathrm{M}_{0}\right)\left(4.10\right.$ ton $\left.\mathrm{ha}^{-1}\right)$ and variety super tropic plus vermicompost $\left(\mathrm{V}_{2} \mathrm{M}_{3}\right)$ applied plots in respect of infested head yield by weight.

Table 4. Mean healthy, infested and total yield of cabbage and percent head infestation (by weight) caused by $S$. litura and $P$. brassicae as influenced by treatment combinations

\begin{tabular}{l|c|c|c|c}
\hline \multirow{2}{*}{$\begin{array}{c}\text { Treatment } \\
\text { combinations }\end{array}$} & \multicolumn{3}{|c|}{ Head yield $\left(\mathrm{t} \mathrm{ha}^{-1}\right)$} & $\begin{array}{c}\text { Percent head } \\
\text { infestation by } \\
\text { weight }\end{array}$ \\
\cline { 2 - 4 } & Healthy & Infested & Total & $10.25 \mathrm{~b}$ \\
$\mathrm{~V}_{1} \mathrm{M}_{0}$ & $53.65 \mathrm{ef}$ & $6.13 \mathrm{~b}$ & $59.78 \mathrm{ef}$ & $6.83 \mathrm{c}$ \\
$\mathrm{V}_{1} \mathrm{M}_{1}$ & $57.60 \mathrm{~d}$ & $4.22 \mathrm{~cd}$ & $61.82 \mathrm{def}$ & $7.20 \mathrm{c}$ \\
$\mathrm{V}_{1} \mathrm{M}_{2}$ & $56.30 \mathrm{de}$ & $4.37 \mathrm{bc}$ & $60.67 \mathrm{ef}$ & $1.84 \mathrm{ef}$ \\
$\mathrm{V}_{1} \mathrm{M}_{3}$ & $69.23 \mathrm{a}$ & $1.30 \mathrm{ef}$ & $70.53 \mathrm{ab}$ & $3.91 \mathrm{de}$ \\
$\mathrm{V}_{1} \mathrm{M}_{4}$ & $64.63 \mathrm{~b}$ & $2.63 \mathrm{cde}$ & $67.26 \mathrm{bc}$ & $6.48 \mathrm{c}$ \\
$\mathrm{V}_{2} \mathrm{M}_{0}$ & $59.20 \mathrm{~cd}$ & $4.10 \mathrm{~cd}$ & $63.30 \mathrm{cde}$ & $6.68 \mathrm{c}$ \\
$\mathrm{V}_{2} \mathrm{M}_{1}$ & $58.78 \mathrm{~cd}$ & $4.21 \mathrm{~cd}$ & $62.99 \mathrm{de}$ & $13.86 \mathrm{a}$ \\
$\mathrm{V}_{2} \mathrm{M}_{2}$ & $50.40 \mathrm{f}$ & $8.11 \mathrm{a}$ & $58.51 \mathrm{f}$ & $4.85 \mathrm{~cd}$ \\
$\mathrm{~V}_{2} \mathrm{M}_{3}$ & $62.13 \mathrm{bc}$ & $3.17 \mathrm{~cd}$ & $65.30 \mathrm{~cd}$ & $0.93 \mathrm{f}$ \\
$\mathrm{V}_{2} \mathrm{M}_{4}$ & $71.47 \mathrm{a}$ & $0.67 \mathrm{f}$ & $72.14 \mathrm{a}$ & 2.512 \\
\hline LSD & 3.614 & 1.827 & 4.143 & \\
\hline$V_{1}$ & & & &
\end{tabular}

$\mathrm{V}_{1}=$ var. Atlas-70; $\mathrm{V}_{2}=$ Super tropic;

$\mathrm{M}_{0}=$ control, $\mathrm{M}_{1}=$ cowdung @ $6 \mathrm{~kg}_{\mathrm{plot}}{ }^{-1}, \mathrm{M}_{2}=$ mustard oilcake @ 750g $\operatorname{plot}^{-1}, \mathrm{M}_{3}=$ Vermicompost @ $3 \mathrm{~kg} \mathrm{plot}^{-1}$ and $\mathrm{M}_{4}=$ Trichoderma @ $3 \mathrm{~kg}_{\mathrm{g}}$ plot $^{-1}$

The total head yield was the highest (72.14 ton ha- ${ }^{-1}$ ) in variety Super tropic and trichoderma $\left(\mathrm{V}_{2} \mathrm{M}_{4}\right)$ applied plots which was statistically similar to $\mathrm{V}_{1} \mathrm{M}_{3}(70.53$ ton $\mathrm{ha}^{-1}$ ) applied plots but significantly higher from all other treatment combinations. On the other hand the lowest total head yield (58.51 ton ha $\left.{ }^{-1}\right)$ was recorded in variety Super tropic and mustard oil cake $\left(\mathrm{V}_{2} \mathrm{M}_{2}\right)$ applied plots followed by variety Atlas- 70 plus control plots $\left(\mathrm{V}_{1} \mathrm{M}_{0}\right)(59.78$ ton/ha) which was 
statistically similar to $\mathrm{V}_{1} \mathrm{M}_{2}$ (60.67 ton ha $\mathrm{a}^{-1}$ ) treated plots. In terms of percent head infestation by weight, the lowest percent $(0.93 \%)$ was recorded in variety Super tropic plus trichoderma $\left(\mathrm{V}_{2} \mathrm{M}_{4}\right)$ applied plots followed by $\mathrm{V}_{1} \mathrm{M}_{3}(1.84 \%)$ treated plots. The highest percent $(13.86 \%)$ head infestation by weight was recorded in variety Super tropic plus mustard oil cake $\left(\mathrm{V}_{2} \mathrm{M}_{2}\right)$ applied plots followed by variety Atlas-70 plus control plots $\left(\mathrm{V}_{1} \mathrm{M}_{0}\right)(10.25 \%)$. No significant difference was observed among variet Atlas-70 plus mustard oil cake $\left(\mathrm{V}_{1} \mathrm{M}_{2}\right)$ (7.20\%), variety Atlas -70 plus cowdung $\left(\mathrm{V}_{1} \mathrm{M}_{1}\right)(6.83 \%)$, variety Super tropic plus cowdung $\left(\mathrm{V}_{2} \mathrm{M}_{1}\right)(6.68 \%)$, variety Super tropic plus control $\left(\mathrm{V}_{2} \mathrm{M}_{0}\right)(6.48 \%)$ in respect of percent infested heads by weight (Table 4).

From the results of Table 4, it is evident that the healthy head weight and total head yield of cabbage was increased in variety Super tropic plus trichoderma $\left(\mathrm{V}_{2} \mathrm{M}_{4}\right)$, and variety Atlas-70 plus vermicompost $\left(\mathrm{V}_{1} \mathrm{M}_{3}\right)$ treated cabbage plots compared to control group and remaining other treatment combinations.

There are many reports highlights the improvement of crop yield by applying vermicompost. Getnet and Raja (2013) observed significant differences in the growth and development of cabbage and pest infestation level between vermicompost applied and control plants. Uptake of soluble phenolic compounds from vermicompost, by the plant tissues makes them unpalatable there by affecting the rates of reproduction and survival of pest (Edwards et al., 2010a; Edwards et al., 2010b). The integration of vermicompost, chemical fertilizer and biofertilizer increased the rice yield by $15.9 \%$ over the use of chemical fertilizer alone (Jeyabal and Kuppusamy, 2001). The garden pea (Pisum sativum) grown by using vermicompost produce higher green pods, higher green grain weight plant $^{-1}$ (Meena et al., 2007). In the present study the cabbage plant grown in vermicompost applied plot received all the essential nutrients there by cabbage head weight was increased significantly compared to untreated control. From the results of the present study, it is evident that the lowest number of infested plant plot $^{-1}$ and lowest percentage of infested leaves plant ${ }^{-1}$ were found in variety Atlas-70 plus varmicompost $\left(\mathrm{V}_{1} \mathrm{M}_{3}\right)$, and variety Super tropic plus trichoderma $\left(\mathrm{V}_{2} \mathrm{M}_{4}\right)$ applied plots. Likewise, the lowest number of infested heads plot ${ }^{-1}$ and lowest percentage of infested head by number and weight, respectively were recoreded for variety Super tropic plus trichoderma $\left(\mathrm{V}_{2} \mathrm{M}_{4}\right)$, and variety Atlas-70 plus vermicompost $\left(\mathrm{V}_{1} \mathrm{M}_{3}\right)$ applied plots. On the other hand, the highest number of healthy heads plot $^{-1}$, highest yield of healthy head were obtained from variety Super tropic plus trichoderma $\left(\mathrm{V}_{2} \mathrm{M}_{4}\right)$ applied plots followed by variety Atlas-70 plus vermicompost $\left(\mathrm{V}_{1} \mathrm{M}_{3}\right)$ applied plots. It is assumed that both varieties have the ability to withstand the infestation caused by $S$. litura and P. brassicae caterpillars. On the other hand, the levels of the infestation on cabbage and the yield of cabbage were also influenced by the application of organic fertilizers. Khan (2007) reported that the variety Atlas-70 showed higher susceptibility to common cutworm in respect of head infestation, it gave higher yield. Khan (2006) reported that the weight of infested and healthy heads varied 
insignificantly by the combined effect of variety and nitrogen fertilizer level. He obtained the highest yield from the treatment combination $\mathrm{V}_{1} \mathrm{~N}_{1}\left(\mathrm{~V}_{1}=\right.$ Variety Atlas-70; $\mathrm{N}_{1}=260 \mathrm{~kg} \mathrm{~N} \mathrm{ha}{ }^{-1}$ ). This might be due to addition of organic amendments that helped in suppressing various insect pests such as European corn borer (Phelan et al., 1996), other corn insect pests (Birader et al., 1998), aphids and scale insects (Huelsman et al., 2000) and brinjal shoot and fruit borer (Sudhakar et al., 1998). Several authors reported that the addition of vermicompost decreased the incidence of S. litura, Helicoverpa armigera, leaf miner (Apoaerema modicella), jassids (Empoasca kerri), aphids (Aphis craccivora) and spider mites on groundnuts (Rao et al., 2001; Rao, 2002; 2003). Significantly resulted lower number of leaf hoppers and thrips in chilli (Ramesh, 2000) and their damage in field crops. Vermicompost substitution to soil reduced the damage to cabbage seedlings by Myzus persicae and cabbage white caterpillars (P. brassicae) (Arancon et al. 2005). Plant grown in inorganic fertilizers is more prone to pest attack than those grown on organic fertilizers (Yardim and Edwards, 2003). Phelan (2004) reported that plant grown with organic fertilizers usually attacked by fewer arthropod pests and can resist pest attack much better than plants received inorganic fertilizers.

Variety Super tropic of cabbage grown in trichoderma $\left(\mathrm{V}_{2} \mathrm{M}_{4}\right)$, and var. Atlas-70 plus vermicompost $\left(V_{1} M_{3}\right)$ applied plots were found to be less infested by $S$. litura and $P$. brassicae in respect of number of infested plants plot $^{-1}$, number and percent of infested leaves plant ${ }^{-1}$ on different dates of observation. The highest healthy heads (by number), healthy head yield (by weight) and total head yield were obtained from combined effect of variety Super tropic plus trichoderma $\left(\mathrm{V}_{2} \mathrm{M}_{4}\right)$ as well as variety Atlas-70 plus vermicompost $\left(\mathrm{V}_{1} \mathrm{M}_{3}\right)$ applied plots while the lowest healthy heads (by number), healthy head yield (by weight) and total head yield were found from variety Super tropic plus mustard oil cake $\left(V_{2} M_{2}\right)$ applied plots as well as variety Atlas-70 plus control plots $\left(\mathrm{V}_{1} \mathrm{M}_{0}\right)$.

\section{References}

Arancon, N. Q., P. A. Galvis and C. A. Edwards. 2005. Suppression of insect pest populations and damage to plants by vermicomposts. Bioresour Technol. 96:11371142.

BBS. 2014. Staistical Pocket Book Bangladesh, Bangladesh Bureau of Statistics. Statistics Division, Ministry of Planning, Government of the People's Republic of Bangladesh, Dhaka. pp. 384.

Bhonwong, A., M. J. Stout, J. Attajarusit and P. Tantasawat. 2009. Defensive role of tomato polyphenoloxidases against cotton bollworm (Helicoverpa armigera) and beet army worm (Spodoptera exigua). J Chem Ecol. 35:28-38

Biradar, A. P., N. D. Sunita, R. G. Teggel and S. B. Devaradavadgi. 1998. Effect of vermicompost on the incidence of subabul psyllid. Insect- Environ 4:55-56

Edwards, C. A., N. Q. Arancon, M. V. Bennett, A. Askar, G. Keeney and B. Little. 2010a. Suppression of green peach aphid (Myzus persicae) (Sulz.), citrus mealybug 
(Planococcus citri) (Risso), and two spotted spider mite (Tetranychus urticae) (Koch.) attacks on tomatoes and cucumbers by aqueous extracts from vermicomposts. Crop. Prot. 29:80-93

Edwards, C. A., N. Q. Arancon, M. V. Bennett, A. Askar and G. Keeney. 2010b. Effect of aqueous extracts from vermicomposts on attacks by cucumber beetles (Acalymna vittatum) (Fabr.) on cucumbers and tobacco hornworm (Manduca sexta) (L.) on tomatoes. Pedobiologia. 53:141-148.

Getnet, M. and N. Raja. 2013. Impact of vermicompost on growth and development of cabbage, Brassica Linn. and their sucking pest, Brevicoryne brassicae Linn. (Homoptera: Aphididae). Res. J. Environ. Earth Sci. 5(3): 104-112.

Huelsman, M. F., C. A. Edwards, J. L. Lawrence and D. O. Clarke-Harris 2000. A study of the effect of soil nitrogen levels on the incidence of insect pests and predators in Jamaican sweet potato (Ipomoea batatus) and Callaloo (Amaranthus). Proc Brighton Pest Control Conference: Pests and Diseases 8D-13:895-900.

Jeyabal, A. and G. Kuppusamy. 2001. Recycling of organic wastes for the production of vermicompost and its response in rice-legume cropping system and soil fertility. Eur. J. Agron. 15(13): 153-170.

Khan, M. M. H. 2006. Influence of variety and nitrogen fertilizer levels on the infestation of the cabbage caterpillar, Pieris Brassicae (L.) (Lepidoptera: Pieridae). Bangladesh J. entomol. 16 (2): 91-98.

Khan, M. M. H. 2007. Field evaluation of some cabbage varieties against common cutworm, Spodoptera litura (F.). J. Bangladesh Agril. Univ. 5 (1): 31-35.

Kumar, P., C. S. Prasad and G. N. Tiwari. 2007. Population intensity of insect pests of cabbage in relation to weather parameters. Ann. Plant. Protect. Sci. 15: 245-246.

Mahanil, S., J. Attajarusit, M. J. Stout and P. Thipayong. 2008. Over expression of tomato phenol oxidase increases resistance to the common cutworm. Plant Sci. 174:456-466.

Mahla, R. S., S. Singh and P. Chaudhary. 2005. Management of diamondback moth, Plutella xylostella (L.) larvae by entomopathogenic fungus, Metarhizium anisopliae. Indian J. Ent. 67: 342-344.

Meena, R. N., Y. Singh, S. P. Singh, J. P. Singh and K. Singh. 2007. Effect of sources and level of organic manure on yield, quality and economics of garden pea (Pisum sativam L.) in eastern uttar pradesh. Vegetable Sci. 34:60-63.

Merrill, M. C. 1983. Eco-agriculture: a review of its history and philosophy. Biol. Agric. Hort. 1: 181-210.

Meyer, G. A. 2000. Interactive effects of soil fertility and herbivory on Brassica nigra. Oikos. 22: 433-441.

Phelan, P. L., K. H. Norris and J. F. Mason. 1996. Soil management history and host preference by Ostrinia nubilatis: evidence for plant mineral balance mediating insectplant interactions. Environ. Entom. 25:1329-1336

Phelan, P. L. 2004. Connecting below-ground and above-ground food webs: the role of organic matter in biological buffering. In: Magadoff F, Well RR (eds) Soil Organic Matter in sustainable agriculture, CRC Press, Boca Raton. pp 199-226. 
Ramesh, P. 2000. Effects of vermicomposts and vermicornposting on damage by sucking pests to ground nut (Arachis hypogea). Indian J. Agri. Sci. 70:334.

Rao, K. R., P. A. Rao and K. T. Rao. 2001. Influence of fertilizers and manures on the population of coccinellid beetles and spiders in groundnut ecosystem. Ann. Plant. Protect. Sci. 9:43-46.

Rao, K. R. 2002. Induce host plant resistance in the management sucking pests of groundnut. Ann. Plant. Protect. Sci. 10:45-50.

Rao, K. R. 2003. Influence of host plant nutrition on the incidence of Spodoptera litura and Helicoverpa armigera on groundnuts. Indian J. Entomol. 65:386-392.

Rao, S. R. K. and O. P. Lal. 2005. Seasonal incidence of mustard aphid, Lipaphis erysimi (Kalt) and diamondback moth, Plutella xylostella (L.) on cabbage. J. Insect Sci. 18(2): 106-110.

Sinha, R. K., S. Agarwal, K. Chaudhan and D. Valani. 2010. The wonders of earthworms and its vermicomposting in farm production: Charles Darwin's friends of farmers', with potential to replace destructive chemical fertilizers from agriculture. Agri. Sci. 1(2): 76-94.

Stevenson, P. C., J. C. Anderson, W. M. Blaney and M. S. J. Simmonds. 1993. Development inhibition of Spodoptera litura (Fab.) larvae by a novel caffeoylquinic acid from the wild groundnut, Arachis paraguariensis (chhod et Hassl.). J. Chem. Ecol. 19: 2917-2933.

Sudhakar, K., K. C. Punnaiah and P. V. Krishnayya. 1998. Influence of organic and inorganic fertilizers and certain insecticides on the incidence of shoot and fruit borer, Leucinodes orbonalis Guen, infesting brinjal. J. Entomol. Res. 22:283-286.

Uddin, M. N., M. M. Hoque, S. M. M. Hossain, S. N. Alam, A. K. M. S. R. Mollik, A. K. M. Khorsheduzzaman, S. Alam, M. M. Rahman, A. N. M. R. Karim, E. G. Rajotte and G. C. Luther. 2007. IPM approach for controlling two lepidopteran pests of cabbage in Bangladesh. Bangladesh J. entomol. 17 (1): 19-29.

Yardim, E. N. and C. A. Edwards. 2003. Effects of organic and synthetic fertilizer sources on pest and predatory insects associated with tomatoes. Phytoparasitica. 31:324-329. 